\title{
Low-frequency oscillations and transport processes induced by multiscale transverse structures in the polar wind outflow: A three-dimensional simulation
}

\author{
Supriya B. Ganguli and Valeriy V. Gavrishchaka \\ Science Applications International Corporation, McLean, Virginia
}

\begin{abstract}
Multiscale transverse structures in the magnetic-field-aligned flows have been frequently observed in the auroral region by FAST and Freja satellites. A number of multiscale processes, such as broadband low-frequency oscillations and various cross-field transport effects are well correlated with these structures. To study these effects, we have used our three-dimensional multifluid model with multiscale transverse inhomogeneities in the initial velocity profile. Self-consistent generation of the low-frequency mode driven by local transverse gradients in the field-aligned ion flow and associated transport processes were simulated. Effects of particle interaction with the self-consistent time-dependent three-dimensional wave potential have been modeled using a distribution of test particles. For typical polar wind conditions it has been found that even large-scale $(\sim 50-100 \mathrm{~km})$ transverse inhomogeneities in the flow can generate low-frequency oscillations that lead to significant flow modifications, cross-field particle diffusion, and other transport effects. It has also been shown that even small-amplitude $(\sim 10-20 \%)$ short-scale $(\sim 10 \mathrm{~km})$ modulations of the original large-scale flow profile significantly increases low-frequency mode generation and associated cross-field transport, not only at the local spatial scales imposed by the modulations but also on global scales. Note that this wave-induced cross-field transport is not included in any of the global numerical models of the ionosphere, ionosphere-thermosphere, or ionosphere-polar wind. The simulation results indicate that the wave-induced cross-field transport not only affects the ion outflow rates but also leads to a significant broadening of particle phase-space distribution and tranverse particle diffusion.
\end{abstract}

\section{Introduction}

A number of in situ observations from OGO 5 , Heos 2, DE 2, and other spacecrafts indicate that large-scale transverse inhomogeneities in the field-aligned currents (FAC) and ion flows are ubiquitous in the ionosphere. The importance of the processes driven by transverse inhomogeneities in the ionospheric plasma flows has been shown both theoretically and from observations [D'Angelo et al., 1974; Potemra et al., 1978; Sugiura et al., 1984; Knudsen et al., 1990; Loranc et al., 1991; Ishii et al., 1992; Horton et al., 1993; Ganguli et al., 1999].

Recent high-resolution data from the Freja [Luhr et al., 1994; Stasiewicz and Potemra, 1998; Knudsen and Wahlund, 1998] and FAST [McFadden et al., 1998; $\mathrm{Pe}$ ria et al., 1998] satellites showed that multiscale structures in the parallel ion flows/beams and FAC with scale lengths from several hundred kilometers down to several hundred meters are a common feature of the auroral 
ionosphere. A number of effects, such as broadband low-frequency oscillations, transverse particle energization, and various multiscale cross-field transport processes, are well correlated with these highly structured parallel flows [Knudsen and Wahlund, 1998; Ergun et al., 1998; McFadden et al., 1998; Amatucci, 1999].

Adequate description of the multiscale processes associated with inhomogeneous parallel flows usually requires three-dimensional (3-D) multimoment and multifluid models. These models use much less compusir resources than the more rigorous kinetic models and include important non-Maxwellian features which are not available in typical magnetohydrodynamic (MHD) global models.

The one-dimensional (1-D) multimoment multifluid models have been successfully applied to a wide range of problems including large-scale modeling of the polar wind [Ganguli et al., 1987; Schunk and Sojka, 1989, 1997], auroral return current regions [Ganguli and Palmadesso, 1987], magnetosphere-ionosphere coupling [Mitchell et al., 1992], and potential structures generation [S.B. Ganguli et al., 1994]. However, because of the 1-D approximation, fluid instabilities due to transverse inhomogeneities in the parallel flows [e.g., D'Angelo, 1965] and cross-field transport effects were ignored.

The first 3-D fluid numerical simulations, which selfconsistently addressed collective effects due to nonuniform parallel flows and their influence on space plasma transport, were performed by Ganguli and Guzdar [1992], Ganguli [1995], and Ganguli et al. [1999]. It was shown that the development of the shear driven instability [D'Angelo, 1965] can have a significant effect on ionospheric plasma transport and saturated states. This initial version of the model considered a uniform magnetic field and also assumed uniform and isotropic temperatures for all species. These studies were mainly focused on the dynamics of the hot magnetospheric plasma with application to the magnetosphere-ionosphere coupling problem.

In this paper we have expanded on our previous work [Ganguli et al., 1999] to address low-frequency instabilities and associated transport effects due to transverse inhomogeneities in a field-aligned flow velocity for a typical low-temperature polar wind plasma. Here we also demonstrate important wave-particle interaction effects through the use of a test particle distribution driven by a time-varying 3-D wave potential. The main focus of this paper is an analytical study and a 3-D simulation of the collective and transport effects due to the multiscale nature of the transverse structures in the parallel flow, as recently observed by the FAST and Freja satellites. 
For this purpose an initial parallel velocity profile with a typical large-scale transverse inhomogeneity modulated by small-scale perturbations of various amplitudes has been used.

The paper is organized as follows. In section 2 we present a model description, the corresponding set of equations, and linear instability criteria. In section 3 we present our analytical and simulation results followed by discussions and conclusions in section 4.

\section{Model Description}

The model is designed to simulate the 3-D dynamics of a fully ionized $\left(e^{-}, \mathrm{H}^{+}\right)$plasma in a flux tube encompassing a magnetic field line. The model is applied to the high-latitude topside ionosphere, and the simulation region extends in altitude from $1500 \mathrm{~km}$ to $\sim 10$ $R_{E}$.

A detailed description of the model and its linear analysis was given by Ganguli et al. [1999] and $\mathrm{Mc}^{-}$ Carthy et al. [1997] and is summarized as follows. The system of 3-D transport equations [Drake et al., 1991] includes continuity and parallel momentum equations for the ions (equations (1) and (2)), an equation for the transverse velocity in the $\mathbf{E} \times \mathbf{B}$ drift approximation (equation (3)), and the relation between the electrostatic potential and plasma density (equation (4)) from the Boltzmann approximation for electrons along the magnetic field,

$$
\begin{gathered}
\frac{\partial n}{\partial t}+\nabla \cdot(n \mathbf{v})=0 \\
\frac{\partial p_{\|}}{\partial t}+\nabla \cdot\left(p_{\|} \mathbf{v}\right)=-\frac{T_{e}+T_{i}}{m} \frac{\partial n}{\partial z} \\
\mathbf{v}_{\perp}=-\frac{\nabla \phi \times \mathbf{B}}{B^{2}} \\
\phi=\frac{T_{e}}{e} \ln \left(\frac{n}{n_{0}}\right) .
\end{gathered}
$$

Here $n$ and $\mathbf{v}$ are ion density and velocity, $p_{i \mid} \equiv n v_{\|}$, $T_{e}$ and $T_{i}$ are electron and ion temperatures, $m$ is ion mass, $\phi$ is electrostatic potential $(E=-\nabla \phi)$, and $\mathbf{B}$ is the magnetic field. The initial equilibrium considered throughout this paper is described by a uniform density $n=n_{0}$, zero potential $\phi=0$, and nonuniform parallel velocity $v(x)$, where $x$ is the transverse coordinate. This is an obvious stationary solution of (1)-(4). Equations (1)-(4) are solved numerically using a trapezoidal 
time-stepping scheme, higher order spatial differencing and hyperviscosity [Guzdar et al., 1993; Ganguli et al., 1999]. The boundary conditions are periodic in parallel $z$ and transverse $y$ directions. Constant values of all moments and zero gradients are maintained at the boundaries in the $x$ direction.

In this simplified version of the model the magnetic field is uniform and the temperature is uniform and isotropic. As discussed in section 4, these assumptions allow us to simplify the interpretation of the effects discussed in this paper, while the essential results obtained here remain valid when more rigorous models are used.

In addition to the basic fluid model used earlier by Gonguli et al. [1999], here we also solve 3-D equations of motion for a distribution of test particles ( $\mathrm{H}^{+}$ions) moving in a 3-D time-dependent self-consistent wave potential. Full particle dynamics is considered along the magnetic field, while a gyrocenter approximation is used in the transverse direction. The gyrocenter approximation saves a significant amount of CPU time since the ion gyroperiod is usually much smaller than a typical time step of fluid simulations. Since only longwavelength low-frequency (much below the ion gyrofrequency) modes are modeled here, the use of the gyrocenter approximation in the transverse direction is an adequate approach to illustrate wave-particle interactions. The equations of motion used for the test particles are given by

$$
\begin{gathered}
M \frac{d^{2} Z}{d t^{2}}=-e \frac{d \phi(X, Y, Z)}{d Z} \\
{\left[\begin{array}{l}
V_{x} \\
V_{y}
\end{array}\right]=\left[\begin{array}{c}
-(\partial \phi / \partial Y) / B \\
(\partial \phi / \partial X) / B
\end{array}\right]}
\end{gathered}
$$

where $X, Y, Z$ and $V_{x}, V_{y}, V_{z}$ are Cartesian particle coordinates and velocity components, respectively. Components of the electrostatic potential gradient $(\nabla \phi)$ are obtained from the numerical solution of the fluid equations (1)-(4) and linearly interpolated to a current particle position $X, Y, Z$. Both sets of equations (1)-(4) and (5) and (6) are solved simultaneously.

First, we present linear results for the D'Angelo instability following D'Angelo [1965] and Catto et al. [1973]. We consider equilibrium with a uniform magnetic field in the $z$ direction, a uniform density, and a nonuniform parallel flow with a gradient $d v_{\| 0} / d x \neq 0$ in the $x$ direction. Considering perturbations proportional to $\exp \left(-i \omega t+i k_{y} y+i k_{z} z\right)$ and linearizing (1)-(4), we obtain the following dispersion relation for the D'Angelo mode in the local approximation:

$$
\omega_{1}^{2}-c_{2}^{2} k_{z}^{2}\left(1-\frac{1}{\Omega_{i}} \frac{d v_{\| 0}}{d x} \frac{k_{v}}{k_{z}}\right)=0
$$


where $\omega_{1} \equiv\left(\omega-k_{z} v_{\| 0}\right)$ is the mode frequency in the ion frame and $c_{\varepsilon} \equiv\left[T_{e}+T_{i} / m\right]^{1 / 2}$ is the sound speed, and $\Omega_{i}$ is the ion cyclotron frequency. A more rigorous version of (7) based on a kinetic formulation is given by G. Ganguli et al. [1994] and Gavrishchaka et al. [1998, 2000].

For uniform parallel flow $\left(d v_{\| 0} / d x=0\right)$, (7) reduces to the conventional dispersion relation for the ionacoustic mode with the solution $\omega_{1}=c_{s} k_{z}$. However, when

$$
\Lambda \equiv\left(\frac{1}{\Omega_{i}} \frac{d v_{\| 0}}{d x} \frac{k_{y}}{k_{z}}\right)>1
$$

the solution of the dispersion relation (7) is an unstable mode with the growth rate

$$
\gamma=c_{s} k_{z}(\Lambda-1)^{1 / 2},
$$

and with a real frequency in the ion frame $\omega_{1 r}=0$. The growth rate maximized over $k_{z}\left(\gamma_{m}\right)$ for a particular value of $k_{y}$ is readily obtained from (9) and is given by

$$
\gamma_{m}=\frac{1}{2} c_{s} k_{y} \frac{d v_{\| 0} / d x}{\Omega_{i}}
$$

Note that although we cannot adequately apply expression (9) and/or use fluid simulations in a range $\left(k_{y} \rho_{i}\right) \geq 1$, we have already shown from a more rigorous kinetic dispersion equation given by $G$. Ganguli et al. [1994] and Gaurishchaka et al. [1998] and by 2D PIC simulations [Gavrishchaka et al., 2000] that the D'Angelo mode is unstable over a wide range of $k_{y}$ (from $\left(k_{y} \rho_{i}\right) \ll 1$ to $\left.\left(k_{y} \rho_{i}\right) \gg 1\right)$. However, as shown by 3-D fluid simulations [McCarthy et al., 1997; Ganguli et al., 1999] and by our 2-D PIC simulations [Gavrishchaka et al., 2000], the long-wavelength modes $\left(k_{y} \rho_{i} \ll 1\right)$ are the main contributors to large scale transport effects. Therefore in the following we consider only effects associated with long-wavelength modes. These are adequately described by our linear (7) and 3-D fluid simulation (1)-(4) models.

In the next section, typical parameters of the polar wind plasma is used to estimate the instability growth rate from (10) for different velocity gradients consistent with observations. Instability development and associated transport effects have been studied using a 3-D numerical model based on equations (1)-(4) and (5) and (6).

\section{Results}

We have investigated plasma collective effects and associated transport phenomena due to transverse inhomogeneities in the field-aligned ion flow. The following

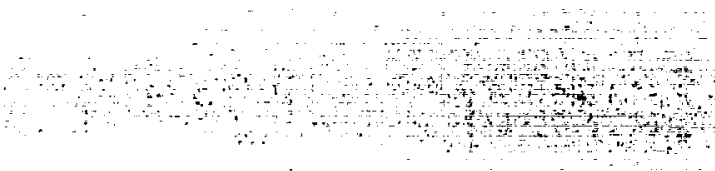


parallel velocity $\left(v_{z}\right)$ profile is used for this study

$$
v_{z}=v_{z 0} \exp \left(-\frac{x^{2}}{L_{t}^{2}}\right)\left[1+\Delta \cos \left(\frac{2 \pi}{\lambda} x\right)\right] .
$$

Here $v_{z 0}$ is the magnitude of the velocity, $L_{t}$ is the transverse scale length, $\Delta$ and $\lambda$ are a small-scale modulation amplitude and scale length, and the magnetic field is in the $z$ direction. When $\Delta=0,(11)$ describes a localized profile with a single transverse scale length $\left(L_{t}\right)$. This case will be used to model flows with relatively lar-2scale transverse inhomogeneities. When $\Delta>0$ and $(\lambda / 2) \ll L_{t},(11)$ describes a filamented multiscale velocity profile, where the average transverse scale length is determined by $L_{t}$, while the local inhomogeneity scale length is determined by $(\lambda / 2)$. The latter case will be used to model multiscale parallel flow profiles established by recent in situ observations [Luhr et al., 1994; Stasiewicz and Potemra, 1998; Knudsen and Wahlund, 1998; McFadden et al., 1998; Ergun et al., 1998; Peria et al., 1998]. Varying $L_{t}, \Delta$, and $\lambda$, we can obtain profiles with different transverse scale lengths, scale separations, degree of filamentation, and local velocity gradients $d v_{z} / d x$.

A profile example with $L_{t}=60 \mathrm{~km}, v_{z 0}=20 \mathrm{~km}$ $\mathrm{s}^{-1}$, and $\Delta=0$ is shown by the solid line in Figure 1a. The same case but with $\Delta=0.2$ and $(\lambda / 2)=10 \mathrm{~km}$ is shown by the dashed line. The corresponding velocity gradient $d v_{z} / d x$ is plotted in Figure $1 \mathrm{~b}$. It is clear that, although the average velocity gradient (determined by $L_{t}$ ) does not change when small-scale modulations are introduced, the local velocity gradients (determined by $\lambda$ and $\Delta$ ) can be significantly increased even by a small amount of small-scale modulation. This is evident from (11), since $d v_{z} / d x$ includes two leading terms: one is $\propto 1 / L_{\ell}$ (average gradient) and the other is $\propto \Delta / \lambda$ (local gradient due to small-scale filamentation). Since the D'Angelo instability is local in nature and is driven by a local velocity gradient $\left(d v_{z} / d x\right)$, the modulation of the initial large-scale flow can have dramatic consequences as will be shown later.

We begin our analysis by estimating the linear growth rates of the D'Angelo instability for a typical polar wind plasma with different transverse scale structures in the parallel velocity profile. The results are presented in Figure 2, where an $(e-, \mathrm{H}+)$ plasma is considered with $T_{e}=1 \mathrm{eV}, T_{H+}=0.2 \mathrm{eV}, \Omega_{H+}=104 \mathrm{rad} \mathrm{s}^{-1}$, $c_{s}=10.7 \mathrm{~km} \mathrm{~s}^{-1}$, and velocity magnitude $v_{z 0}=20 \mathrm{~km}$ $\mathrm{s}^{-1}$. In Figure 2a we plot the growth rate, optimized in $k_{z}$, given by (10) for different values of the transverse localization $L_{t}$. We use the maximum value of $d v_{z} / d x$ as obtained from (11) for $\Delta=0$ (i.e., no modulation). Here $k_{y}=1.14 \times 10^{-4} \mathrm{~m}^{-1}$ (i.e., $\lambda_{y}=5.5 \times 10^{4} \mathrm{~m}$ ),

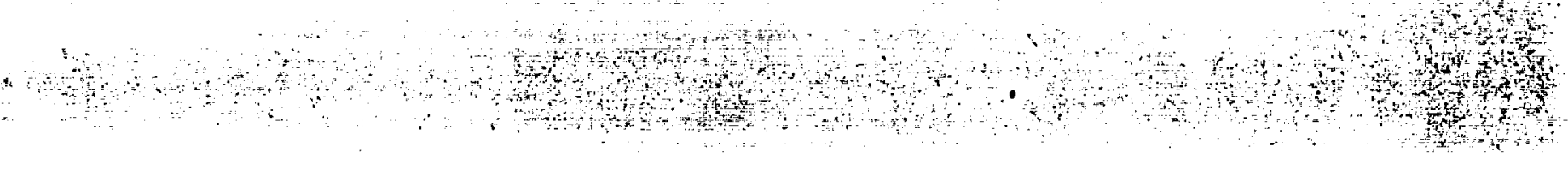


which will be used later in our 3-D simulations. One can see that even for large transverse scale sizes $(\sim 50-100$ $\mathrm{km}$ ) the growth rate of the D'Angelo instability is significantly large.

A more important feature is the growth rate sensitivity to the transverse inhomogeneity scale length. As seen from Figure 2a, a 2 orders of magnitude reduction in the transverse scale length leads to an almost 2 orders of magnitude increase in the growth rate of the D'Angelo instability. Thus highly localized flow profiles can be a very effective source of the low-frequency instability and associated transport effects.

However, an isolated highly localized flow filament is not the most interesting flow configuration. First, it is not the most natural configuration encountered in the real space environment. Second, all collective and transport effects induced by this localized profile will occur in a small spatial region determined by the filament transverse scale leading to minimal effects on the global plasma dynamics. A more natural configuration confirmed by observations [e.g., Stasiewicz and Potemra, 1998; Peria et al., 1998] is a multiscale flow and/or FAC profile with multiple transverse structures of different scale. As shown later, this configuration is capable of inducing significant large-scale transport effects in addition to localized processes. Equation (11) models such a profile with two different scales and multiple filaments. In the more natural general case instead of one filament scale, one can use a statistical distribution of different filament scales. However, to illustrate the main effect and for an easier theoretical interpretation, (11) is an appropriate choice.

Now we use (11) with $v_{z 0}=20 \mathrm{~km} \mathrm{~s}^{-1}, L_{t}=60 \mathrm{~km}$, and various modulation (filament) amplitudes $(\Delta)$, and scale lengths $(\lambda)$. The maximum local velocity gradients $\left(d v_{z} / d x\right)$ obtained from differentiation of (1i) are shown in Figure $2 b$ as a function of $\Delta$ for $(\lambda / 2)$ equal to 20,10 , 5 , and $1 \mathrm{~km}$ (solid, dashed, dotted-dashed, and dotted lines, respectively). We see a significant increase in the local velocity gradient when short-scale modulations, even with small amplitude, are introduced.

Since the growth rate of the D'Angelo instability is determined by the local velocity gradient (shear frequency), the maximum growth rate of the instability (optimized over $k_{z}$ ) is readily obtained from (10) by using $d v_{z} / d x$ from Figure $2 \mathrm{~b}$. The resulting growth rate for modulation scales used in Figure $2 b$ is shown as a function of $\Delta$ in Figure 2c. As in Figure 2a, we use $k_{y}=1.14 \times 10^{-4} \mathrm{~m}^{-1}$. It is clear that even smallamplitude short-scale modulations can lead to a significant increase in the instability growth rate. 
Short-scale modulations lead not only to the growth rate increase for a particular transverse wave number but also to a significant broadening of the unstable range in $k_{y}$ (or more generally $k_{\perp}$ ) space. As was discussed by Gavrishchaka et al. [2000], the wide $k_{y}$ range of the simultaneously unstable modes leads to a broadband continuous wave spectrum when even a small transverse electric field $\left(E_{x}\right)$ is introduced. This effect is due to differences in frequency Doppler shifts ( $k_{y} V_{E}$, where $V_{E}$ is the $E \times B$ drift velocity) experiencad by the modes with different $k_{y}$. A transverse $\mathrm{DC}$ electric field is often observed in space [e.g., Amatucci, 1999, and references therein]. The D'Angelo mode can be unstable over a wide range of $k_{y}$, especially in the presence of the short-scale filamentation. Therefore the broadband low-frequency wave activity observed in correlation with multiscale parallel flows and ion beams [e.g., Ergun et al., 1998] can be at least in part explained by the described mechanism.

We have shown that even small-amplitude short-scale modulation (filamentation) of the initial large-scale fieldaligned flow can lead to a significant increase in the effective growth rate of the D'Angelo instability. This is due to the increase in the local velocity gradient. However, a linear calculation cannot show how small-scale filamentation of the initial steady state will change the overall instability development and associated transport effects. Linear analysis is also unable to clarify whether local enhancements of the instability and associated transport processes will lead to a significant change in the global plasma behavior on scales large compared with the filament (modulation) scale. To answer all these questions, we use our 3-D fluid model (1)-(4) with a distribution of test particles moving in a time-dependent 3-D wave potential according to (5) and (6).

We start our simulations of the polar wind $(e-, \mathrm{H}+)$ plasma with $T_{i}=0.2 \mathrm{eV}, T_{e}=1 \mathrm{eV}$, and an initial flow profile given by (11). Initially, small density perturbations $\propto \sin \left(k_{y} y+k_{z} z\right)$ are introduced to seed the instability. The following flow parameters are used: $v_{z 0}=20$ $\mathrm{km} \mathrm{s}^{-1}, L_{t}=30 \mathrm{~km}$, and $(\lambda / 2)=10 \mathrm{~km}$. The modulation amplitude $(\Delta)$ is varied to study the effects of short-scale filamentation. We use a set of wave numbers $k_{y}=1.14 \times 10^{-4} \mathrm{~m}^{-1}$ and $k_{z}=9.91 \times 10^{-8} \mathrm{~m}^{-1}$ (i.e., $\lambda_{y}=5.5 \times 10^{4} \mathrm{~m}$ and $\lambda_{z}=6.34 \times 10^{7} \mathrm{~m}$ ). Even though single-mode seeding limits the number of unstable modes, it allows us to clearly illustrate all the important effects and facilitates the unambiguous interpretation of the results. Simulations with random phase multiple mode seeding (not shown in this pa- 
per) confirm all the main results presented here. In the following we compare instability development and associated transport effects induced by inhomogeneous parallel flow both with $(\Delta>0)$ and without $(\Delta=0)$ short-scale filamentation.

In Figure 3a we present the time dependence of the wave potential amplitude for $\Delta=0,0.1,0.2$, and 0.3 (solid, dashed, dot-dashed, and dotted lines, respectively). It is clear from this figure that even smallamplitude short-scale filamentation significantly accierates the plasma response to a structured field-aligned flow. Note, however, that the saturated state is relatively independent of $\Delta$.

As suggested by the linear analysis presented earlier, the main effects of short-scale modulation are due to the increase of the local velocity gradient leading to enhancement of the D'Angelo instability growth rate. This is confirmed by our simulations as shown in Figures $3 \mathrm{~b}$ and $3 \mathrm{c}$. Figure $3 \mathrm{~b}$ presents the maximum velocity gradient of the initial profile (11) as a function of $\Delta$. The growth rate obtained from the simulation curves shown in Figure 3a is given in Figure 3c by a solid line. This growth rate is equal to a $1 / 2$ slope of the straight line that provides the best fit to the upper envelope of the curve $\ln \phi_{\max }^{2}(t)$ in the linear regime. The dashed line represents the linear growth rate obtained from (9) using the $d v_{z} / d x$ values given in Figure $3 \mathrm{~b}$. It is clear that the $\Delta$ dependence of the growth rate obtained from simulations is close to that of the linear growth rate and is consistent with the $\Delta$ dependence of the maximum velocity gradient. The difference in the absolute values of the analytical linear growth rate and that obtained from the simulation may be due to artificial diffusion and viscosity terms used in the simulations for numerical stability. The artificial dissipation terms provide stabilization of short-scale fluctuations in -the simulation system to compensate for the absence of natural (kinetic) dissipative effects in the fluid approximation [Guzdar et al., 1993; Ganguli et al., 1999; Gavrishchaka et al., 1999]. The high-order numerical scheme used here [Zalesak, 1981; Guzdar et al., 1993] allows also to avoid excessive numerical diffusion typical for usual upwind differencing methods [e.g., Press et al., 1992].

In Figures $4 \mathrm{a}-4 \mathrm{e}$ the dynamics of the parallel velocity profiles (averaged over $y$ ) for $\Delta=0$ (solid line) and $\Delta=0.2$ (dashed line) are compared. The profiles are given for $t=0$ (Figure 4a), $1774 \mathrm{~s}$ (Figure 4b), $2129 \mathrm{~s}$ (Figure 4c), $2838 \mathrm{~s}$ (Figure 4d), and $3193 \mathrm{~s}$ (Figure 4e). We see that even small-amplitude initial short-scale filamentation of the profile leads to significant differences in the subsequent flow evolution due to the D'Angelo 
instability. Figure $4 \mathrm{f}$ presents both profiles in the saturated state (solid line, $t=4258 \mathrm{~s}$; dashed line, $t=3193$ s). This figure illustrates that saturated states for both initial loadings are quite similar.

Figure 4 also clearly illustrates the stabilization mechanism of the D'Angelo instability. Instability development broadens velocity profile decreasing velocity shear down to subcritical value which leads to saturation. In the absence of the explicit sources of dissipation, the spatially averaged velocity in the simulation box :mains to be constant. On the other hand, there is a tangeable decrease in the average kinetic energy of the parallel flow due to wave generation. In the presented simulation the change in the average velocity between initial and saturated state was less than $1 \%$ (in both cases), while the change in the average kinetic energy was $\sim 16 \%$ (without filamentation) and $\sim 22 \%$ (with filamentation).

So far we have presented only fluid diagnostics from our simulation. We find that in some respects the dynamics of the test particles gives a better illustration of the effects induced by short-scale filamentation. In Figure 5 we compare the dynamics of the test particles' phase space $\left(V_{z}, X\right)$ for the same parameters and time as in Figures $4 \mathrm{a}-4 \mathrm{e}$. The cases with $\Delta=0$ and $\Delta=0.2$ are shown in the left and right columns, respectively. Initially, all particles are located at the plane $z=0$ and have velocities corresponding to the local fluid velocity. All figures with particles presented in this paper illustrate the integrated picture of all particles from all $z$ and/or $y$ planes. One can clearly see that in the case of short-scale initial filamentation, particle diffusion in phase space begins significantly earlier and is much more intense compared to the case without shortscale modulation. We also see that at earlier times diffusion is localized and clearly correlates with the maxima of the local velocity gradients (see Figure $5 \mathrm{~b}$ ) in accordance with the local nature of the D'Angelo instability. However, at later times transverse mixing begins (Figure 5c) and, finally, the initial small-scale structure is completely destroyed and only large-scale transverse diffusion is evident (Figures $5 \mathrm{~d}$ and $5 \mathrm{e}$ ). Thus initial small-scale filamentation not only increases local crossfield transport but, more importantly, global transport.

Similar effects are evident from test particle dynamics in configuration space $(x, y)$ presented in Figure 6. As in Figure 5, cases with $\Delta=0$ and $\Delta=0.2$ are shown in left and right columns, respectively. The time for Figures $6 \mathrm{~b}-6 \mathrm{e}$ is the same as for Figure $5 \mathrm{~b}-5 \mathrm{e}$. In Figure $6 \mathrm{a}$ we present a snapshot at $t=1419 \mathrm{~s}$. For $t=0$ there is a simple uniform distribution. Again, we 
see that particle diffusion starts earlier and is signifcantly more effective in the case with initial short-scale filamentation. The local nature of the transverse diffusion is clear at early times (Figures $6 \mathrm{a}$ and $6 \mathrm{~b}$ ). Later diffusion becomes global and no sign of the initial smallscale structure remains (Figures 6c-6e). It is interesting to note that both phase space and configuration space evolutions of the test particle distribution (Figures 5 and 6) are qualitatively very similar to those observed in a fully kinetic 2-D PIC simulation of the D'Angeso instability [Gourishchaka et al., 2000].

Finally, in Plates 1 and 2 we present the time evolution of the 3-D velocity profile for $\Delta=0$ and $\Delta=0.2$, respectively. Color represents velocity in $\mathrm{km} \mathrm{s}^{-1}$. Snapshots in Plate 1 are given at $t=0$ (Plate 1a), $3193 \mathrm{~s}$ (Plate 1b), $3903 \mathrm{~s}$ (Plate 1c), $4967 \mathrm{~s}$ (Plate 1d), and in Plate 2 at $t=0$ (Plate 2a), $2129 \mathrm{~s}$ (Plate 2b), 2838 $\mathrm{s}$ (Plate 2c). In both cases we observe significant flow modifications as the instability develops. The maximum flow velocity drops by $\sim 35 \%(\sim 50 \%)$ from its initial value for $\Delta=0(\Delta=0.2)$. Comparisons of Plates 1 and 2 (i.e., $\Delta=0$ and $\Delta=0.2$ ) reveal that not only does the initial filamentation accelerate instability development but it also leads to development of finer spatial structures, even though only single-wave perturbation was used. The latter is mainly due to phase mixing of the modes excited in different local velocity gradient regions created by the initial short-scale modulations in the velocity profile.

\section{Discussion and Conclusions}

We have presented results from the application of our 3-D multimoment, multifluid model to a typical polar wind outflow with multiscale transverse structures in a field-aligned ion flow. The 3-D set of fluid equations allowed a self-consistent simulation of the low-frequency D'Angelo mode driven by the transverse gradient in the parallel velocity and its effects on transport processes in linear and nonlinear stages. We have also solved the equations of motion for a large ensemble of test particles driven by the 3-D time-dependent wave potential obtained from the fluid model. This important addition to a fluid model allowed us to demonstrate waveparticle interaction effects and particle transport due to low-frequency shear-driven waves.

Our linear analysis and simulations show that for typical polar wind conditions $\left(T_{i}=0.2 \mathrm{eV}, T_{e}=1 \mathrm{eV}\right.$, $\left.V_{\|}=20 \mathrm{~km} \mathrm{~s}^{-1}\right)$ even a large-scale $\left(L_{\perp} \sim 100 \mathrm{~km}\right)$ transverse inhomogeneity in a parallel flow velocity can trigger the low-frequency D'Angelo instability with a typical growth time $1 / \gamma \sim 3 \mathrm{~min}$. This is a rather con-
Plates 1 and 2 
servative estimate, since only the long-wavelength limit has been considered $\left(\lambda_{y}>50 \mathrm{~km}\right)$. The growth time is smaller for shorter wavelength. We also find that instability development leads to a significant modification in the flow (maximum velocity changes by $>30 \%$ ) due to induced cross-field plasma transport. Analysis of test particle dynamics reveals that the growth of the D'Angelo mode also leads to substantial particle diffusion in phase space and configuration space. The latter results are qualitatively consistent with typical fedtures observed in more rigorous but small-scale kinetic 2-D PIC simulations of the D'Angelo instability [Gavrishchaka et al., 2000].

The main focus of this paper has been a study of the effects associated with multi-scale transverse structures in parallel flows. We found that even small-amplitude $(10-20 \%)$ short-scale $(\sim 1-10 \mathrm{~km})$ filamentation of the parallel flow leads to significant acceleration of the lowfrequency D'Angelo instability (which depends on local velocity gradient values) and associated transport effects. More importantly, we have shown that short-scale filamentation leads not only to local transport enhancement on the scales determined by filamentation but also significant transport intensification on the global scale.

Our results are relevant to the interpretation of the recent high-resolution FAST and Freja satellite observations of the transversely localized multiscale flows and currents correlated with broadband low-frequency wave activity and transverse transport effects [McFadden et al., 1998; Ergun et al., 1998; Knudsen and Wahlund, 1998; Stasiewicz and Potemra, 1998; Peria et al., 1998]. The reported results are especially topical since in many cases observed field-aligned currents are too small [e.g., Amatucci et al., 1994; Amatucci, 1999, and references therein] to explain low-frequency wave generation by current-driven mechanisms [Kindel and Kènnel, 1971]. On the other hand, we have shown here and recently by kinetic linear analysis and PIC simulations [Gavrishchaka et al., 2000] that the D'Angelo instability can lead to a broadband continuous wave spectrum and a rich set of associated transport effects without any fieldaligned current.

In the present study we used a simplified model with a uniform magnetic field and an isotropic plasma temperature to provide a simple and clear illustration of all the important effects. Preliminary results from the more complete model, which includes a dipole magnetic field and an anisotropic ion temperature, shows that for typical parameters used in this paper the D'Angelo instability can be simultaneously operational over a wide range of altitudes (several $R_{E}$ ) [Ganguli and Gavrishchaka, 
2000]. Therefore we expect that conclusions presented here will not change in any major way when the more rigorous model is applied. The complete set of the quantitative results from the model with dipole magnetic field and anisotropic temperature [Gavrishchaka et al., 1999] will be presented in a future article.

It should be also noted that in the presented simulation quite conservative parameters have been used which resulted in the instability growth time $\gamma^{-1}$ up to $10 \mathrm{~min}$. As indicated by our linear analysis (e.g., Figure 2), the growth time for typically observed plasma parameters can easily be in the range of several seconds or less.

The instability analysis and simulation presented in this paper have been restricted to $\mathrm{H}+$ plasma with one ion component. In reality, space plasma is multi-ion. For example, at lower altitudes it is $(\mathrm{O}+, \mathrm{H}+)$ plasma where $\mathrm{O}+$ is a dominant component. Although the detailed study of the D'Angelo instability in the multicomponent plasma is beyond the scope of this paper, we can summarize our preliminary results on this subject obtained from a full kinetic dispersion relation [Gavrishchaka et al., 1998, 2000] generalized to two-ion component plasma as follows.

The effect of the addition of the $\mathrm{O}+$ component to the $\mathrm{H}+$ plasma critically depends on the shear in the parallel velocity of the $\mathrm{O}+$ component. For example, when $\mathrm{O}+$ component is added to the $\mathrm{H}+$ plasma and $\mathrm{O}+$ flow is shearless, then the growth rate of the D'Angelo instability due to the shear in the $\mathrm{H}+$ flow slowly decreases as $\mathrm{O}+$ concentration increases. However, even in a plasma with $80-90 \%$ of $\mathrm{O}+$ small shear in the $\mathrm{H}+$ flow $\left(d v_{z} / d x \ll \Omega_{H+}\right)$ can still excite the D'Angelo mode. On the other hand, when the shears in the $\mathrm{O}+$ and $\mathrm{H}+$ flows are comparable, even a small addition of $\mathrm{O}+$ can lead to a dramatic increase in thè instability growth rate, since the value $\left(d v_{z} / d x\right) / \Omega_{\mathrm{O}_{+}}$, which enters dispersion relation, is much larger for $\mathrm{O}+$ than that for $\mathrm{H}+$. Further research on this subject is warranted.

Acknowledgments. We thank P. N. Guzdar for useful discussions. This work is supported by the National Aeronautics and Space Administration and the National Science Foundation.

Michel Blanc thanks Pierre-Louis Blelly and Shigeto Watanabe for their assistance in evaluating this paper.

\section{References}

Amatucci, W.E., Inhomogeneous plasma flows: A review of in situ observations and laboratory experiments, J. Geophys. Res., 104, 14481, 1999.

Amatucci, W.E., M.E. Koepke, J.J. Carroll III, and T.E. Sheridan, Observation of ion-cyclotron turbulence at small 
values of magnetic-field-aligned current, Geophys. Res. Lett., 21, 1595, 1994.

Catto, P.J., M.N. Rosenbluth, and C.S. Liu, Parallel velocity shear instabilities in an inhomogeneous plasma with a sheared magnetic field, Phys. Fluids, 16, 1719, 1973.

D'Angelo, N., Kelvin-Helmholtz instability in a fully ionized plasma in a magnetic field, Phys. Fluids, 8, 1748, 1965.

D'Angelo, N., A. Bahnsen, and H. Rosenbauer, Wave and particle measurements at the polar cusp, J. Geophys. Res., 79, 3129, 1974.

Drake, J.F., P.N. Guzdar, and A. Dimits, Three-dimensional simulation of $\nabla T_{i}$-driven turbulence and transport, $P$ hỳs. Fluids $B$, S, 1937, 1991.

Ergun, R.E., et al., FAST satellite observations of electric field structures in the auroral zone, Geophys. Res. Lett., 25, 2025, 1998.

Ganguli, G., M.J. Keskinen, H. Romero, R. Heelis, T. Moore, and C. Pollock, Coupling of microprocesses and macroprocesses due to velocity shear: An application to the low-altitude ionosphere, J. Geophys. Res., 99, 8873, 1994.

Ganguli, S.B., Coupling of micro- and mesoscale processes in the polar wind plasma transport: A generalized fluid model with microprocesses, in Cross-Scale Coupling in Space Plasmas, Geophys. Monogr. Ser., vol. 93, edited by J.L. Horwitz, N. Singh, and J.L. Burch, p. 141, AGU, Washington, D. C., 1995.

Ganguli, S.B., and V.V. Gavrishchaka, Low-frequency turbulence and cross-field transport due to inhomogeneous parallel flows with multiple spatial scales: 3-D simulations, Eos Trans., $A G U, 81(19)$, Spring Meet. Suppl., SM51A-02, 2000.

Ganguli, S.B., and P.N. Guzdar, Three-dimensional plasma transport in planetary magnetospheres, paper presented at 3rd Huntsville workshop on M/I plasma models, 1992.

Ganguli, S.B., and P.J. Palmadesso, Plasma transport in the auroral return current region, J. Geophys. Res., 92, $8673,1987$.

Ganguli, S.B., H.G. Mitchell, and P.J. Palmadesso, Behaviour of ionized plasma in the high latitude topside ionosphere: The polar wind, Planet. Space Sci., 35, 703, 1987.

Ganguli, S.B., H.G. Mitchell, and P.J. Palmadesso, Auroral plasma transport processes in the presence of $\mathrm{kV}$ potential structures, J. Geophys. Res., 99, 5761, 1994.

Ganguli, S.B., P.N. Guzdar, V.V. Gavrishchaka,

W.A. Krueger, and P.E. Blanchard, Cross-field transport due to low frequency oscillations in the auroral region: A three-dimensional simulation, J. Geophys. Res., 104, 4297, 1999.

Gavrishchaka, V.V., S.B. Ganguli, and G.I. Ganguli, Origin of low-frequency oscillations in the ionosphere, Phys. Rev. Lett., 80, 728, 1998.

Gavrishchaka, V.V., S.B. Ganguli, and P.N. Guzdar, Threedimensional simulations of the ionospheric plasma transport in the presence of the structured field-aligned flows, J. Geophys. Res., 104, 22511, 1999.

Gavrishchaka, V.V., G.I. Ganguli, W.A. Scales, S.P. Slinker, C.C. Chaston, J.P. McFadden, R.E. Ergun, and C.W. Carlson, Multi-scale coherent structures and broadband waves due to parallel inhomogeneous flows, Phys. Rev. Lett., 85, 4285, 2000. 
Guzdar, P.N., J.F. Drake, D. McCarthy, A.B. Hassam, and C.S. Liu, Three-dimensional fluid simulations of the nonlinear drift-resistive ballooning modes in tokamak edge plasmas, Phys. Fluids B, 5, 3712, 1993.

Horton, W., J.Q. Dong, X.N. Su, and T. Tajima, Ion mixing in the plasma sheet boundary layer by drift instabilities, J. Geophys. Res., 98, 13,377, 1993.

Ishii, M., M. Sugiura, T. Iyemori, and J. Slavin, Correlation between magnetic and electric field perturbations in the field-aligned current regions deduced from DE-2, $J$. Geophys. Res., 97, 13,877, 1992.

Knudsen, D.J., and J.-E. Wahlund, Core ion flux bursis within solitary kinetic Alfven waves, J. Geophys. Res., 103, 4157, 1998.

Knudsen, D.J., M.C. Kelley, G.D. Earle, J.F. Vickrey, and M. Boehm, Distinguishing Alfven waves from quasi-static field structures associated with discrete aurora, Geophys. Res. Lett., 17, 921, 1990.

Loranc, M., W.B. Hanson, R.A. Heelis, and J.-P. St.-Maurice, A morphological study of vertical ionospheric flows in the high-latitude $F$ region, J. Geophys. Res., 96, 3627, 1991.

Luhr, H., J. Warnecke, L.J. Zanetti, P.A. Lindqvist, and T.J. Hughes, Fine structure of field-aligned current sheets deduced from spacecraft and ground-based observations: Initial FREJA results, Geophys. Res. Lett., 28, 1883, 1994.

McCarthy, D.R., A.E. Booth, J.F. Drake, and P.N. Guzdar, Three-dimensional simulations of the parallel velocity shear instability, Phys. Plasmas, \&, 300, 1997.

McFadden, J.P., et al., Spatial structure and gradients of ion beams observed by FAST, Geophys. Res. Lett., 25, $2021,1998$.

Mitchell, H.G., S.B. Ganguli, and P.J. Palmadesso, Diodelike response of high-latitude plasma in M-I coupling in the presence of field-aligned currents, J. Geophys. Res., 97, 12,045, 1992.

Peria, W.J., C.W. Carlson, J.W. Bonnell, and R.C. Elphic, A statistical study of field-aligned currents, using a database of FAST measurements, Eos Trans. AGU, 79(17), Spring Meet. Suppl., S301, 1998.

Potemra, T.A., J.P. Doering, W.K. Peterson, C.O. Bostrom, R.A. Hoffman, and L.H. Brace, AE-C observations of lowenergy particles and ionospheric temperatures in the turbulent polar cusp: Evidence for the Kelvin-Helmholtz instability, J. Geophys. Res., 83, 3877, 1978.

Press, W.H., S.A. Teukolsky, W.T. Vetterling, and B.P. Flannery, Numerical Recipes, The Art of Scientific Computing, Cambridge Univ. Press, New York, 1992.

Schunk, R.W., and J.J. Sojka, A three-dimensional timedependent model of the polar wind, J. Geophys. Res., 94, 8973, 1989.

Schunk, R.W., and J.J. Sojka, The global ionosphere-polar wind system during changing magnetic activity, $J$. Geophys. Res., 102, 11,625, 1997.

Stasiewicz, K., and T. Potemra, Multiscale current structures observed by Freja, J. Geophys. Res., 103, 4315, 1998.

Sugiura, M., T. Iyemori, R.A. Hoffman, N.C. Maynard, J.L. Burch, and J.D. Winningham, Relationships between field-aligned currents, electric fields, and particle precipitation as observed by DE-2, in Magnetospheric Currents, Geophys. Monogr. Ser., vol. 38, edited by T.A. Potemra,

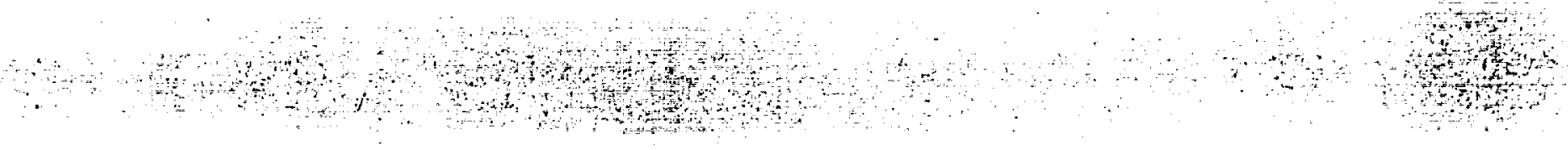


Figure 1. (a) Velocity profile $v_{z}(x)$ and (b) corresponding velocity gradient $d v_{z} / d x$ obtained from (11) for $L_{t}=60 \mathrm{~km},(\lambda / 2)=10 \mathrm{~km}, v_{z 0}=20 \mathrm{~km} \mathrm{~s}^{-1}, \Delta=0$ (solid line), and $\Delta=0.2$ (dashed line).

Figure 1. (a) Velocity profile $v_{z}(x)$ and (b) corresponding velocity gradient $d v_{z} / d x$ obtained from (11) for $L_{t}=60 \mathrm{~km},(\lambda / 2)=10 \mathrm{~km}, v_{z 0}=20 \mathrm{~km} \mathrm{~s}^{-1}, \Delta=0$ (solid line), and $\Delta=0.2$ (dashed line).

Figure 2. (a) Linear growth rate, optimized in $k_{z}$ (equation (10)), for a typical polar: wind plasma as a function of transverse scale length in the parallel velocity profile. Here an $(e-, \mathrm{H}+)$ plasma is considered with $T_{e}=1 \mathrm{eV}, T_{H+}=0.2 \mathrm{eV}, \Omega_{H+}=104 \mathrm{rad}$ $\mathrm{s}^{-1}, c_{\mathrm{s}}=10.7 \mathrm{~km} \mathrm{~s}^{-\top}, k_{y}=1.14 \times 10^{-4} \mathrm{~m}^{-1}$, and velocity magnitude $v_{z 0}=20 \mathrm{~km} \mathrm{~s}^{-1}$. The maximum value of $d v_{z} / d x$ obtained from (11) for $\Delta=0$ is used. (b) The maximum local velocity gradient $\left(d v_{z} / d x\right)$ obtained from differentiation of (11) as a function of $\Delta$ for $L_{t}=60 \mathrm{~km}$ and $(\lambda / 2)$ equal to 20,$10 ; 5$, and 1 $\mathrm{km}$ (solid, dashed, dotted-dashed, and dotted lines, respectively). Other parameters are same as in Figure 2a. (c) The growth rate (optimized over $k_{z}$ ) obtained from (11) for modulation scales used (Figure $2 b$ ) as a function of $\Delta$. The values of $d v_{z} / d x$ from Figure $2 \mathrm{~b}$ are used. Other parameters are same as in Figure 2a and Figure 2b.

Figure 2. (a) Linear growth rate, optimized in $k_{z}$ (equation (10)), for a typical polar wind plasma as a function of transverse scale length in the parallel velocity profile. Here an $(e-, \mathrm{H}+)$ plasma is considered with $T_{e}=1 \mathrm{eV}, T_{H+}=0.2 \mathrm{eV}, \Omega_{H+}=104 \mathrm{rad} \mathrm{s}^{-1}, c_{s}=10.7 \mathrm{~km} \mathrm{~s}^{-1}$, $k_{y}=1.14 \times 10^{-4} \mathrm{~m}^{-1}$, and velocity magnitude $v_{z 0}=20 \mathrm{~km} \mathrm{~s}^{-1}$. The maximum value of $d v_{z} / d x$ obtained from (11) for $\Delta=0$ is used. (b) The maximum local velocity gradient $\left(d v_{z} / d x\right)$ obtained from differentiation of (11) as a function of $\Delta$ for $L_{t}=60 \mathrm{~km}$ and $(\lambda / 2)$ equal to $20,10,5$, and $1 \mathrm{~km}$ (solid, dashed, dotted-dashed, and dotted lines, respectively). Other parameters are same as in Figure 2a. (c) The growth rate (optimized over $k_{z}$ ) obtained from (11) for modulation scales used (Figure $2 \mathrm{~b}$ ) as a function of $\Delta$. The values of $d v_{z} / d x$ from Figure $2 \mathrm{~b}$ are used. Other parameters are same as in Figure $2 \mathrm{a}$ and Figure $2 \mathrm{~b}$.

Figure 3. Results of the 3-D simulations of the polar wind $(e-, \mathrm{H}+)$ plasma with $T_{i}=0.2 \mathrm{eV}, T_{\mathrm{e}}=1 \mathrm{eV}$, and an initial flow profile given by (11). Initially, small density perturbations $\propto \sin \left(k_{y} y+k_{z} z\right)$ are introduced to seed the instability. The following flow parameters are used: $v_{z 0}=20 \mathrm{~km} \mathrm{~s}^{-1}, L_{t}=30 \mathrm{~km}$, and $(\lambda / 2)=10$ $\mathrm{km}$. The modulation amplitude $(\Delta)$ is varied to study the effects of short-scale filamentation. One set of wave numbers $k_{y}=1.14 \times 10^{-4} \mathrm{~m}^{-1}$ and $k_{z}=9.91 \times 10^{-8}$ $\mathrm{m}^{-1}$ (i.e., $\lambda_{y}=5.5 \times 10^{4} \mathrm{~m}$ and $\lambda_{z}=6.34 \times 10^{7} \mathrm{~m}$ ) is used. (a) Time dependence of the maximum wave potential amplitude for $\Delta=0,0.1,0.2$, and 0.3 (solid, dashed, dot-dashed, and dotted lines, respectively). (b) Maximum velocity gradient of the initial profile (11) as a function of $\Delta$. (c) Growth rate obtained from the simulation curves (solid line) shown in Figure $3 \mathrm{a}$ and the corresponding linear growth rate obtained from (9) for simulation parameters (dashed line) as a function of $\Delta$. 
Figure 3. Results of the 3-D simulations of the polar wind (e-, $\mathrm{H}+)$ plasma with $T_{\mathrm{i}}=0.2$ $\mathrm{eV}, T_{e}=1 \mathrm{eV}$, and an initial flow profile given by (11). Initially, small density perturbations $\propto \sin \left(k_{y} y+k_{z} z\right)$ are introduced to seed the instability. The following flow parameters are used: $v_{z 0}=20 \mathrm{~km} \mathrm{~s}^{-1}, L_{t}=30 \mathrm{~km}$, and $(\lambda / 2)=10 \mathrm{~km}$. The modulation amplitude $(\Delta)$ is varied to study the effects of short-scale filamentation. One set of wave numbers $k_{y}=1.14 \times 10^{-4} \mathrm{~m}^{-1}$ and $k_{z}=9.91 \times 10^{-8} \mathrm{~m}^{-1}$ (i.e., $\lambda_{y}=5.5 \times 10^{4} \mathrm{~m}$ and $\lambda_{z}=6.34 \times 10^{7} \mathrm{~m}$ ) is used. (a) Time dependence of the maximum wave potential amplitude for $\Delta=0,0.1,0.2$, and 0.3 (solid, dashed, dot-dashed, and dotted lines, respectively). (b) Maximum velocity gradient of the initial profile (11) as a function of $\Delta$. (c) Growth rate obtained from the simulation curves (solid line) shown in Figure $3 \mathrm{a}$ and the corresponding linear growth rate obtained from (9) for simulation parameters (dashed line) as a function of $\Delta$.

Figure 4. Dynamics of the parallel velocity profiles (averaged over $y$ ) obtained from 3-D simulation for $\Delta=$ 0 (solid line) and $\Delta=0.2$ (dashed line). The profiles are given for (a) $t=0$, (b) $1774 \mathrm{~s}$, (c) $2129 \mathrm{~s}$, (d) $2838 \mathrm{~s}$, and (e) $3193 \mathrm{~s}$. (f) Both profiles are shown in saturated state (solid line $-t=4258 \mathrm{~s}$, dashed line $-t=3193 \mathrm{~s}$ ). Other simulation parameters are same as in Figure 3.

Figure 4. Dynamics of the parallel velocity profiles (averaged over $y$ ) obtained from 3-D simulation for $\Delta=0$ (solid line) and $\Delta=0.2$ (dashed line). The profiles are given for (a) $t=0$, (b) $1774 \mathrm{~s}$, (c) $2129 \mathrm{~s}$, (d) $2838 \mathrm{~s}$, and (e) $3193 \mathrm{~s}$. (f) Both profiles are shown in saturated state (solid line $-t=4258 \mathrm{~s}$, dashed line $-t=3193 \mathrm{~s}$ ). Other simulation parameters are same as in Figure 3.

Figure 5. Comparison of the dynamics of the test particles' phase space $\left(V_{z}, X\right)$ for the same simulation parameters and time as in Figures $4 \mathrm{a}-4 \mathrm{e}$. The cases with $\Delta=0$ and $\Delta=0.2$ are shown in the left and right columns, respectively.

Figure 5. Comparison of the dynamics of the test particles' phase space $\left(V_{z}, X\right)$ for the same simulation parameters and time as in Figures $4 a-4 e$. The cases with $\Delta=0$ and $\Delta=0.2$ are shown in the left and right columns, respectively.

Figure 6. Test particle dynamics in configuration space $(x, y)$ for the same simulation as in Figures 4 and 5. Cases with $\Delta=0$ and $\Delta=0.2$ are shown in left and right columns, respectively. The time for Figures $6 \mathrm{~b}-6 \mathrm{e}$ is the same as for Figures $5 \mathrm{~b}-5 \mathrm{e}$. In Figure $6 \mathrm{a} a$ snapshot at $t=1419 \mathrm{~s}$ is shown. For $t=0$ there is a simple uniform distribution.

Figure 6. Test particle dynamics in configuration space $(x, y)$ for the same simulation as in Figures 4 and 5 . Cases with $\Delta=0$ and $\Delta=0.2$ are shown in left and right columns, respectively. The time for Figures $6 \mathrm{~b}-6 \mathrm{e}$ is the same as for Figures $5 \mathrm{~b}-5 \mathrm{e}$. In Figure 6a a snapshot at $t=1419$ $\mathrm{s}$ is shown. For $t=0$ there is a simple uniform distribution.

Plate 1. Time evolution of the 3-D velocity profile obtained from simulation. Color represents velocity in $\mathrm{km} \mathrm{s}^{-1}$. Here $\Delta=0$ and other simulation parameters are same as in Figures 3-6. Snapshots are given at (a) $t=0$, (b) $3193 \mathrm{~s}$, (c) $3903 \mathrm{~s}$, and (d) $4967 \mathrm{~s}$.

Plate 1. Time evolution of the 3-D velocity profile obtained from simulation. Color represents velocity in $\mathrm{km} \mathrm{s}^{-1}$. Here $\Delta=0$ and other simulation parameters are same as in Figures 3-6. Snapshots are given at (a) $t=0$, (b) $3193 \mathrm{~s}$, (c) $3903 \mathrm{~s}$, and (d) $4967 \mathrm{~s}$. 
Plate 2. Time evolution of the 3-D velocity profile obtained from simulation. Color represents velocity in $\mathrm{km} \mathrm{s}^{-1}$. Here $\Delta=0.2$ and other simulation parameters are same as in Plate 1. Snapshots are given at (a) $t=$ 0 , (b) $2129 \mathrm{~s}$, and (c) $2838 \mathrm{~s}$.

Plate 2. Time evolution of the 3-D velocity profile obtained from simulation. Color represents velocity in $\mathrm{km} \mathrm{s}^{-1}$. Here $\Delta=0.2$ and other simulation parameters are same as in Plate 1. Snapshots are given at (a) $t=0$, (b) $2129 \mathrm{~s}$, and (c) $2838 \mathrm{~s}$. 


\section{Supplemental Abstract forJGR-Space Physics Manuscripts}

2001JA900023.

Multiscale transverse structures in the magnetic-fieldaligned flows have been frequently observed in the auroral region by FAST and Freja satellites. A number of multiscale processes, such as broadband low-frequency oscillations and various cross-field transport effects are well correlated with these structures. To study these effects, we have used our three-dimensional multifluid model with multiscale transverse inhomogeneities in the initial velocity profile. Self-consistent generation of the low-frequency mode driven by local transverse gradients in the feld-aligned ion flow and associated transport processes were simulated. Effects of particle interaction with the self-consistent timedependent three-dimensional wave potential have been modeled using a distribution of test particles. For typical polar wind conditions it has been found that even large-scale $(\sim 50-100 \mathrm{~km})$ transverse inhomogeneities in the flow can generate low-frequency oscillations that lead to significant flow modifications, cross-field particle diffusion, and other transport effects. It has also been shown that even smallamplitude $(\sim 10-20 \%)$ short-scale $(\sim 10 \mathrm{~km})$ modulations of the original large-scale flow profile significantly increases low-frequency mode generation and associated cross-field transport, not only at the local spatial scales imposed by the modulations but also on global scales. Note that this wave-induced cross-field transport is not included in any of the global numerical models of the ionosphere, ionospherethermosphere, or ionosphere-polar wind. The simulation results indicate that the wave-induced cross-field transport not only affects the ion outflow rates but also leads to a significant broadening of particle phase-space distribution and tranverse particle diffusion. 
GANGULI AND GAVRISHCHAKA: MULTI-SCALE CROSS-FIELD TRANSPORT GANGULI AND GAVRISHCHAKA: MULTI-SCALE CROSS-FIELD TRANSPORT GANGULI AND GAVRISHCHAKA: MULTI-SCALE CROSS-FIELD TRANSPORT GANGULI AND GAVRISHCHAKA: MULTI-SCALE CROSS-FIELD TRANSPORT GANGULI AND GAVRISHCHAKA: MULTI-SCALE CROSS-FIELD TRANSPORT GANGULI AND GAVRISHCHAKA: MULTI-SCALE CROSS-FIELD TRANSPORT GANGULI AND GAVRISHCHAKA: MULTI-SCALE CROSS-FIELD TRANSPORT GANGULI AND GAVRISHCHAKA: MULTI-SCALE CROSS-FIELD TRANSPORT GANGULI AND GAVRISHCHAKA: MULTI-SCALE CROSS-FIELD TRANSPORT GANGULI AND GAVRISHCHAKA: MULTI-SCALE CROSS-FIELD TRANSPORT GANGULI AND GAVRISHCHAKA: MULTI-SCALE CROSS-FIELD TRANSPORT GANGULI AND GAVRISHCHAKA: MULTI-SCALE CROSS-FIELD TRANSPORT GANGULI AND GAVRISHCHAKA: MULTI-SCALE CROSS-FIELD TRANSPORT GANGULI AND GAVRISHCHAKA: MULTI-SCALE CROSS-FIELD TRANSPORT GANGULI AND GAVRISHCHAKA: MULTI-SCALE CROSS-FIELD TRANSPORT GANGULI AND GAVRISHCHAKA: MULTI-SCALE CROSS-FIELD TRANSPORT GANGULI AND GAVRISHCHAKA: MULTI-SCALE CROSS-FIELD TRANSPORT GANGULI AND GAVRISHCHAKA: MULTI-SCALE CROSS-FIELD TRANSPORT GANGULI AND GAVRISHCHAKA: MULTI-SCALE CROSS-FIELD TRANSPORT GANGULI AND GAVRISHCHAKA: MULTI-SCALE CROSS-FIELD TRANSPORT GANGULI AND GAVRISHCHAKA: MULTI-SCALE CROSS-FIELD TRANSPORT GANGULI AND GAVRISHCHAKA: MULTI-SCALE CROSS-FIELD TRANSPORT GANGULI AND GAVRISHCHAKA: MULTI-SCALE CROSS-FIELD TRANSPORT GANGULI AND GAVRISHCHAKA: MULTI-SCALE CROSS-FIELD TRANSPORT GANGULI AND GAVRISHCHAKA: MULTI-SCALE CROSS-FIELD TRANSPORT 
GANGULI AND GAVRISHCHAKA: MULTI-SCALE CROSS-FIELD TRANSPORT GANGULI AND GAVRISHCHAKA: MULTI-SCALE CROSS-FIELD TRANSPORT GANGULI AND GAVRISHCHAKA: MULTI-SCALE CROSS-FIELD TRANSPORT GANGULI AND GAVRISHCHAKA: MULTI-SCALE CROSS-FIELD TRANSPORT GANGULI AND GAVRISHCHAKA: MULTI-SCALE CROSS-FIELD TRANSPORT GANGULI AND GAVRISHCHAKA: MULTI-SCALE CROSS-FIELD TRANSPORT GANGULI AND GAVRISHCHAKA: MULTI-SCALE CROSS-FIELD TRANSPORT GANGULI AND GAVRISHCHAKA: MULTI-SCALE CROSS-FIELD TRANSPORT GANGULI AND GAVRISHCHAKA: MULTI-SCALE CROSS-FIELD TRANSPORT GANGULI AND GAVRISHCHAKA: MULTI-SCALE CROSS-FIELD TRANSPORT GANGULI AND GAVRISHCHAKA: MULTI-SCALE CROSS-FIELD TRANSPORT GANGULI AND GAVRISHCHAKA: MULTI-SCALE CROSS-FIELD TRANSPORT GANGULI AND GAVRISHCHAKA: MULTI-SCALE CROSS-FIELD TRANSPORT GANGULI AND GAVRISHCHAKA: MULTI-SCALE CROSS-FIELD TRANSPORT GANGULI AND GAVRISHCHAKA: MULTI-SCALE CROSS-FIELD TRANSPORT GANGULI AND GAVRISHCHAKA: MULTI-SCALE CROSS-FIELD TRANSPORT GANGULI AND GAVRISHCHAKA: MULTI-SCALE CROSS-FIELD TRANSPORT 

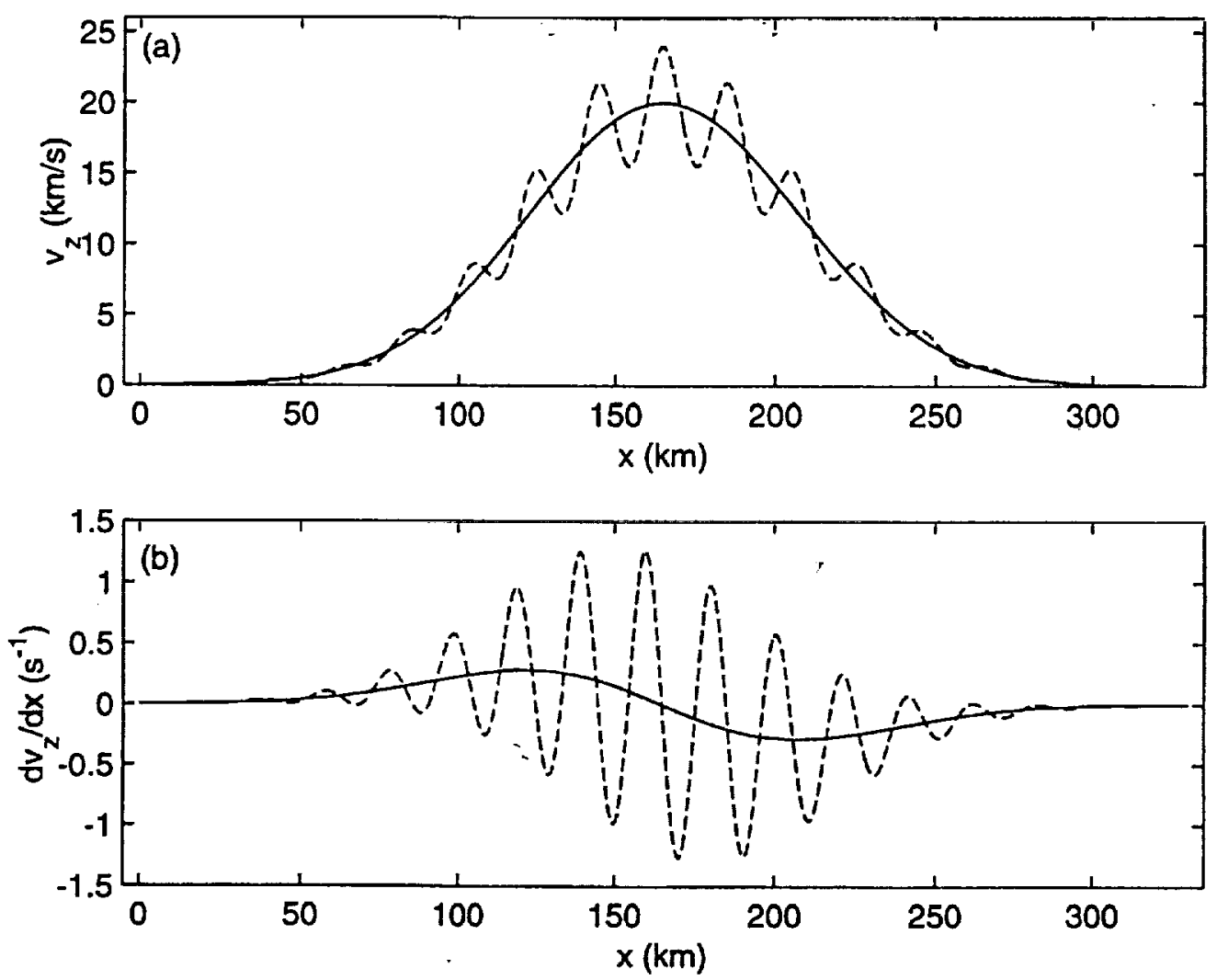
$-$
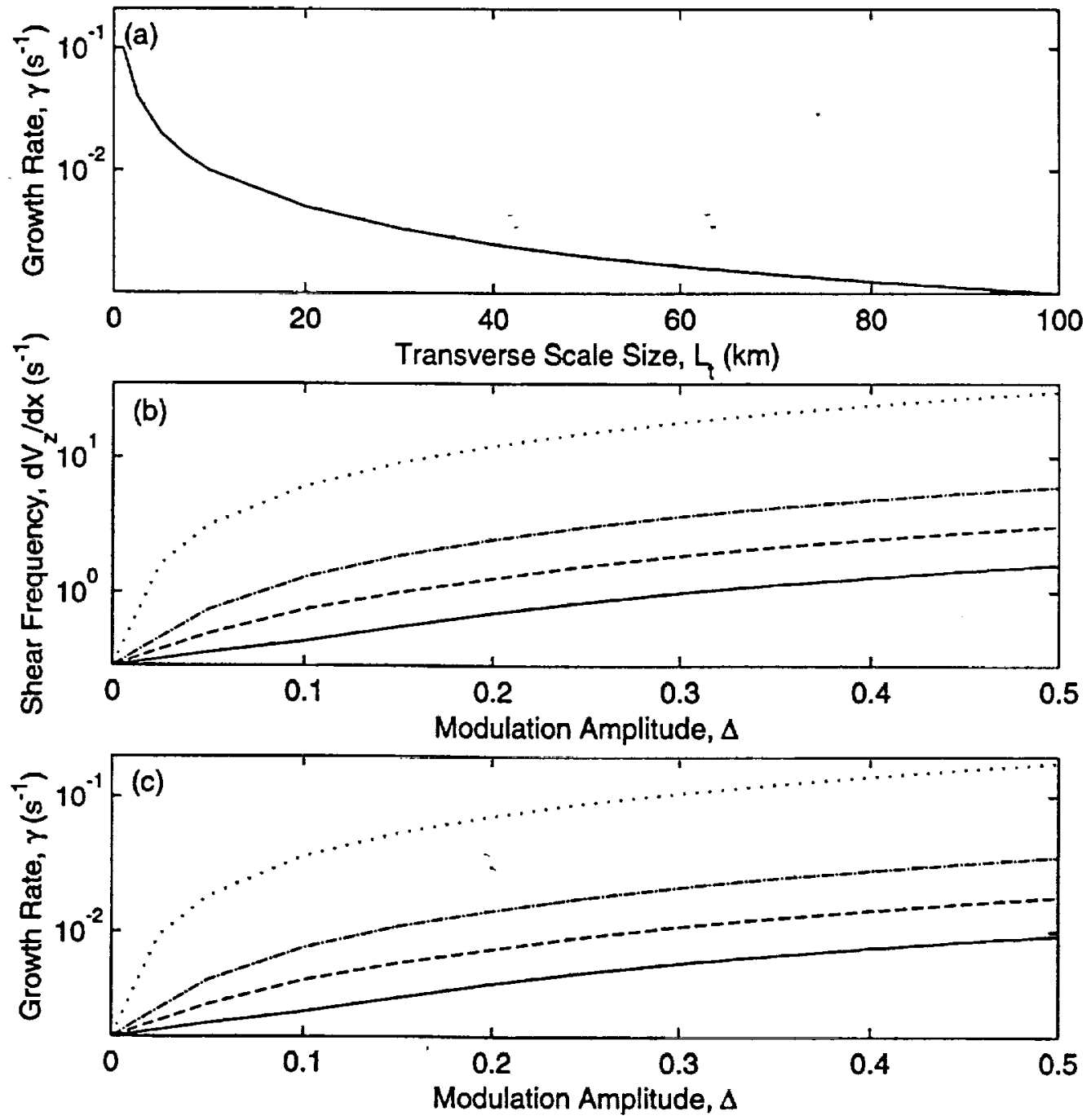

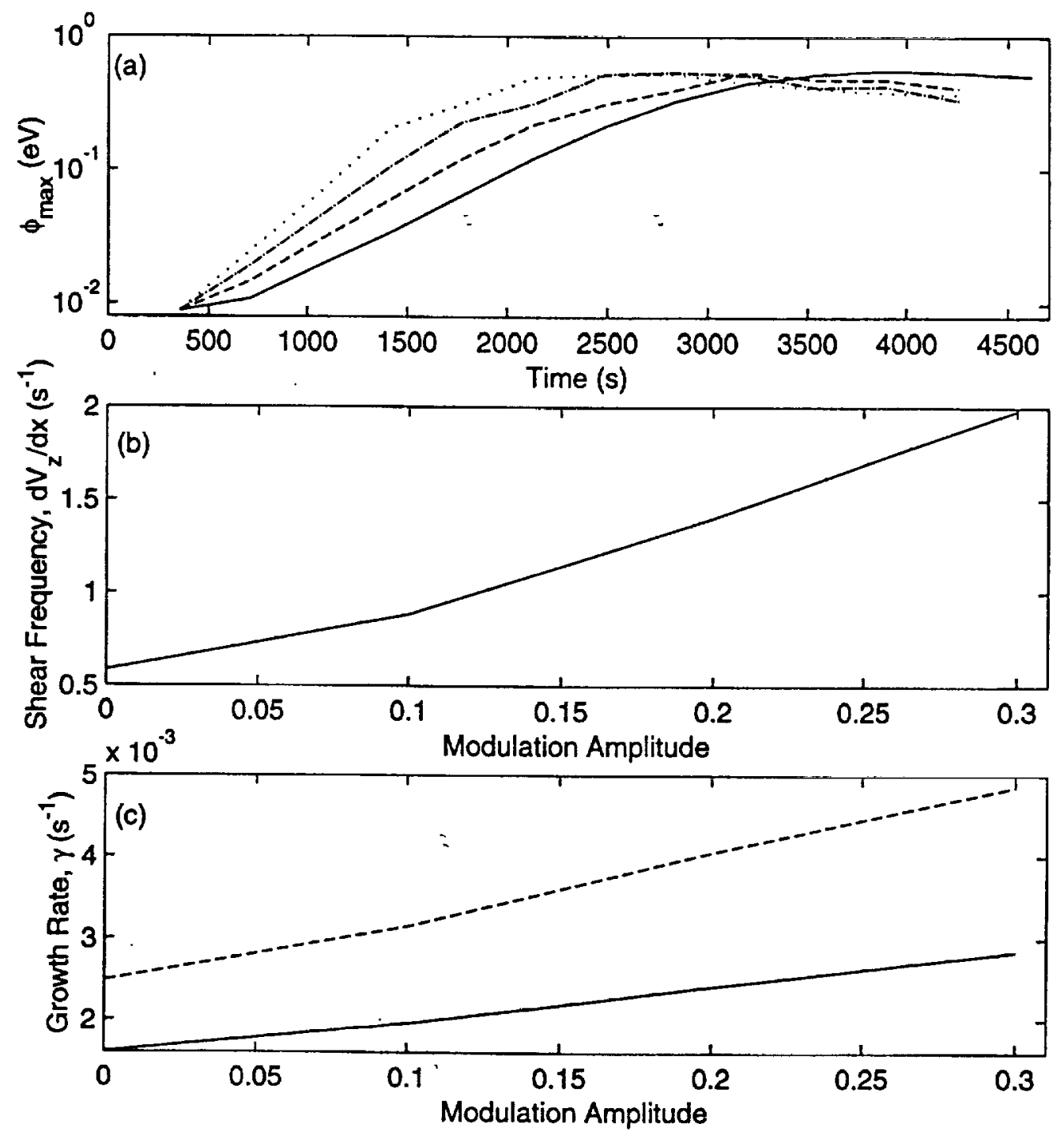

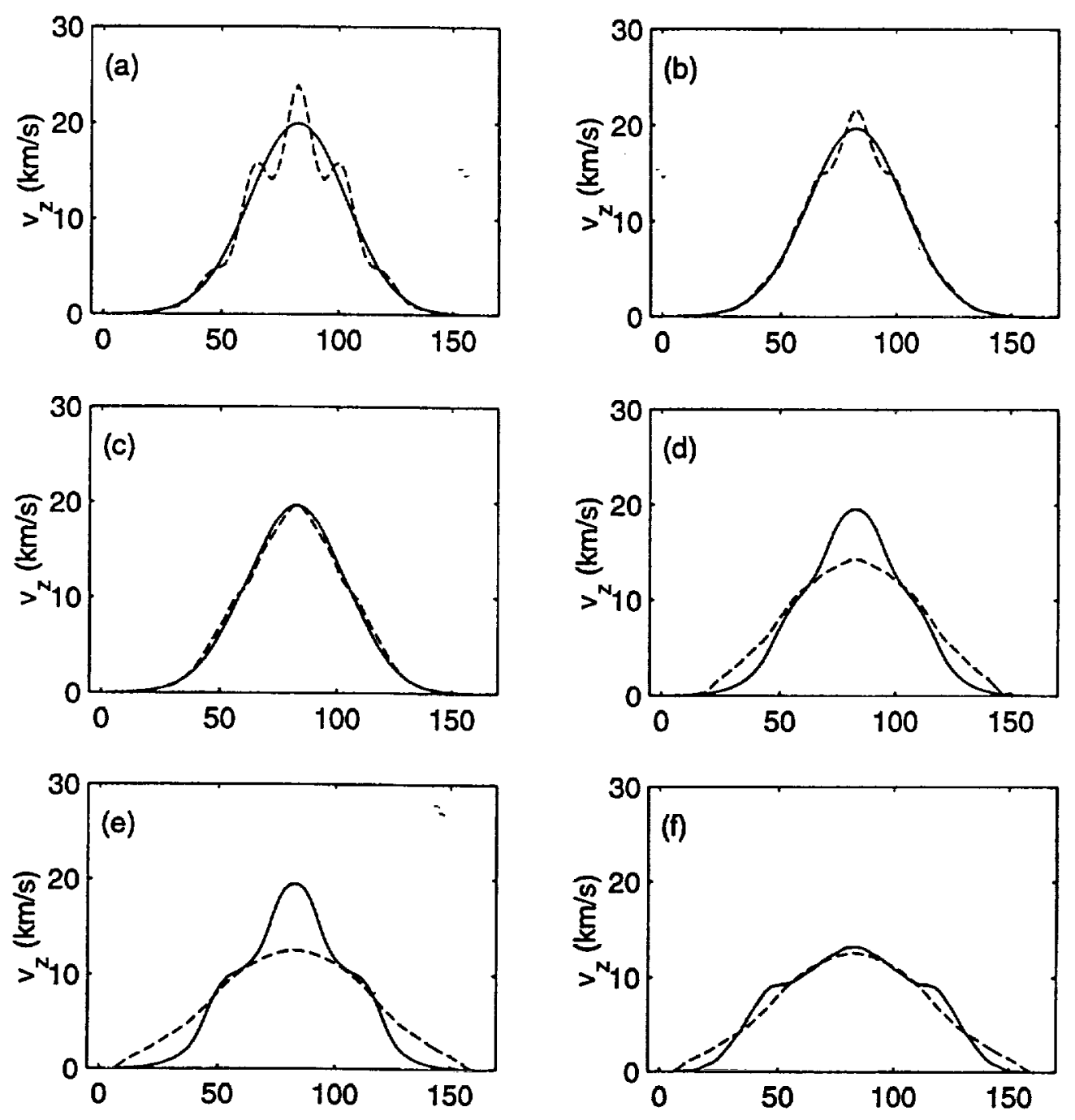

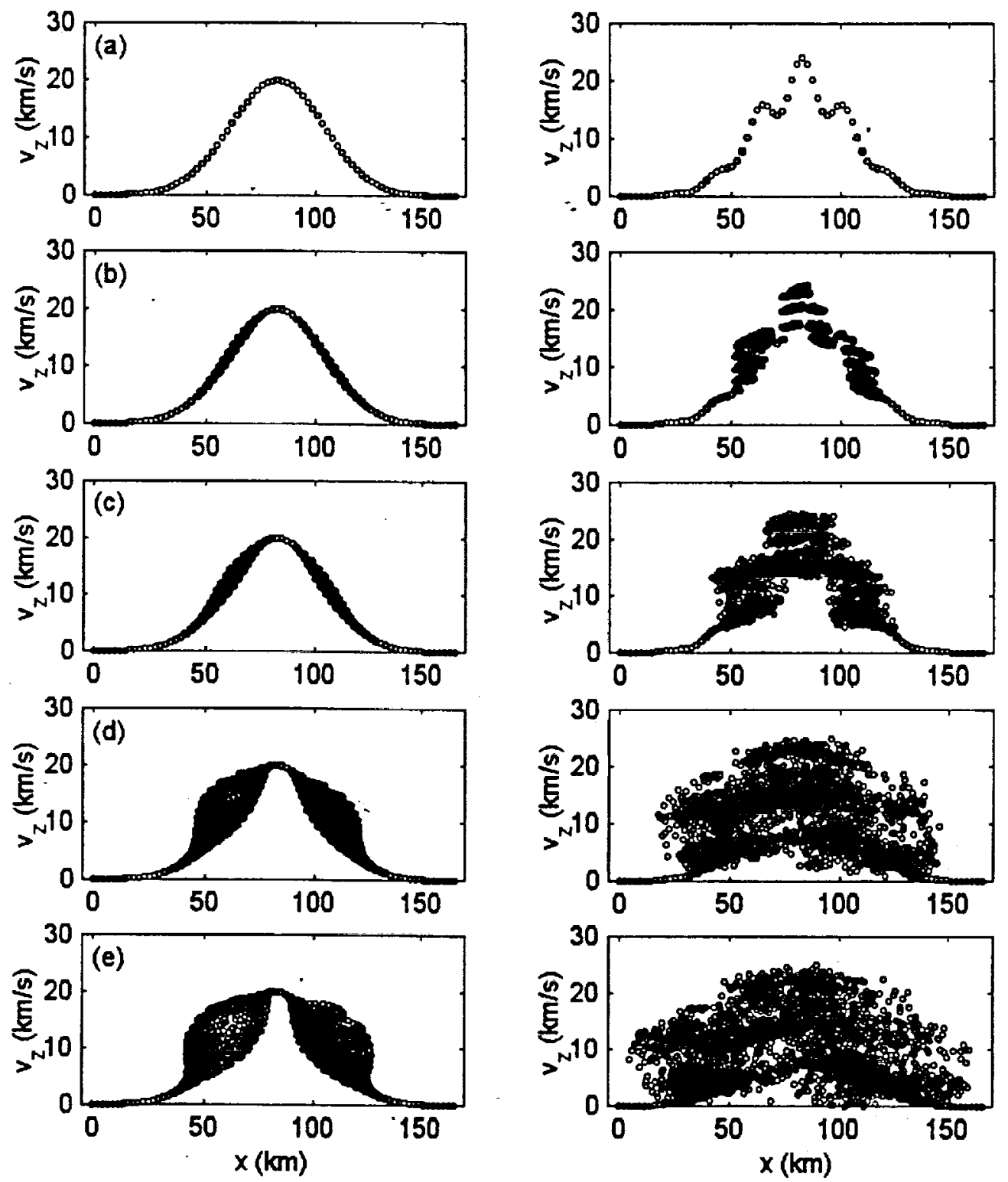

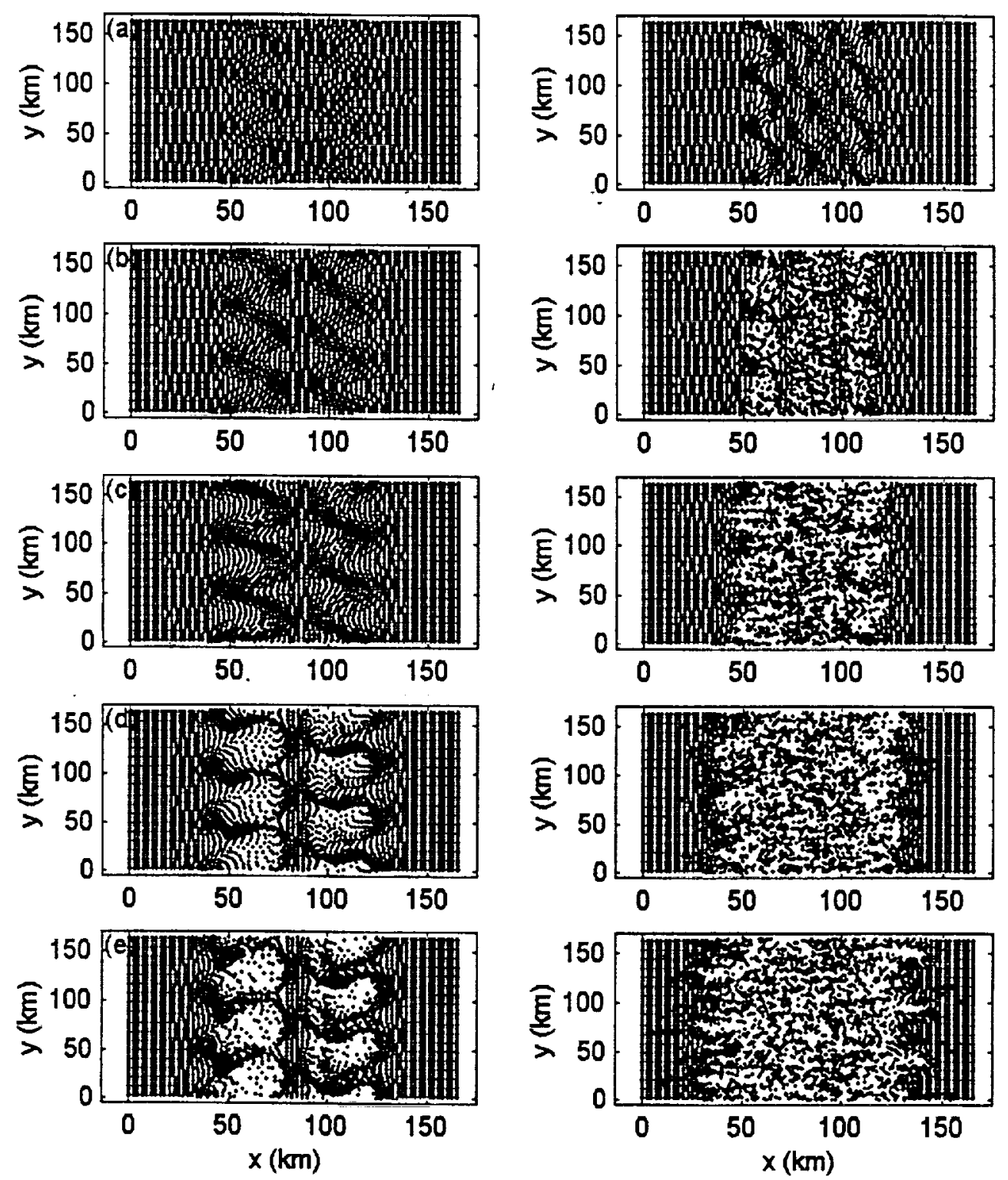